\title{
EFFECT OF OCCLUSAL-CONVERGENCE AND RESIN CEMENT ON RETENTION OF CUBIC-ZIRCONIA BASED CROWNS
}

\author{
Nermeen Nagi Saad* and Maged Zohdy**
}

\begin{abstract}
Purpose. The purpose of this study was to evaluate the retention of cubic zirconia crowns with two occlusal convergence preparations and cementation with two resin cements.

Materials and methods. 28 dies were divided according to the convergence angles into two main groups. Group (1): $20^{\circ}(\mathrm{n}=14)$ Group (2): $12^{\circ}(\mathrm{n}=14)$ Each group was subdivided according to resin cement used into two subgroups. Subgroup A: RelyX Unicem (3M/ESPE, USA) $(n=7)$ Subgroup B: Panavia v5 with clearfil primer plus (Kurary, Japan) $(n=7)$ Two stainless steel master dies were prepared by a milling machine to simulate all ceramic full coverage crown preparation of a maxillary premolar. Each Steel die was machined with height $5.5 \mathrm{~mm}$ and two planes occlusal reduction with palatal cusp bevel. The external diameter of the cylinder was $7.5 \mathrm{~mm}$. one die was prepared with $12^{\circ}$ and the other with $20^{\circ}$ occlusal convergence angle. Impressions of the main master dies were taken and poured using a non-shrink epoxy resin material. The crowns were cemented. Instron universal testing machine was used to test the retention. The cemented crowns were pulled off along the path of insertion with a crosshead speed of $0.5 \mathrm{~mm} /$ minute. The forces required for dislodgment of the crowns were recorded in N. Data were statistically analyzed using one-way ANOVA and many comparisons were made using .Tukey's test at $\mathrm{p}<0.05$.
\end{abstract}

Results. Retention of the zirconia crowns was significantly affected by the occlusal convergence and by the type of resin cement. Self-etching resin cement showed a higher significant result in comparison to self-adhesive. Retention values with occlusal convergence $12^{\circ}$ was highly significant with both cements

Conclusions. The Retention of cubic zirconia ceramics varied significantly depending on the type of resin cement and degree of occlusal convergence of the preparation.

* Lecturer, Department of Fixed Prosthodontics, Alfayoum University, Alfayoum, Egypt

** Assistant Professor, Fixed Prosthodontics Department, Ain Shams University 


\section{INTRODUCTION}

Fabricating all-ceramic restorations with minimal or no application of a secondary phase while maintaining esthetics has been a sought-after goal of the dental profession. The objective has been development of a monolithic material with optical properties similar to the natural tooth structure without the need for porcelain layering. ${ }^{(1)}$ Zirconia is a metastable material that can exist in many crystalline phases, three types of which have been utilized for dental work: tetragonal, monoclinic, and cubic. The first version of zirconia employed in dentistry is a form comprised of the high-strength tetragonal crystalline phase and it's still in use for more than ten years. At room temperature zirconia exists in the weaker monoclinic crystalline form; however, small amounts of oxides, or dopants, are added to stabilize the tetragonal and cubic crystalline forms. ${ }^{(1)}$

The original high-strength zirconia, while having excellent physical properties and a white hue, is opaque and requires layering with porcelain in the same fashion as a porcelain-fused-to-metal restoration to obtain even reasonable esthetics ${ }^{\text {(2) }}$

In an attempt to improve monolithic ceramics with acceptable translucency, processing of the next generation of 3Y-TZPs was refined largely by drastically reducing the concentration of alumina additive and eliminating porosity by sintering at a higher temperature. This led to modest improvement in translucency. ${ }^{(3)}$ While suitable for monolithic posterior restorations, these secondgeneration zirconias were still insufficiently aesthetic for use as monoliths in the anterior zone but at least paved the way to further improvements. The next stage in monolithic zirconia development came with a move to include some transparent phase in the final product to reduce opacity. This was achieved by using a higher yttria content to produce partially stabilized zirconias, $4 \mathrm{~mol} \%$
(4YPSZ) or $5 \mathrm{~mol} \%$ (5Y-PSZ), with increased amounts of nonbirefringent $\mathrm{c}$ phase. This markedly improved translucency, but strength and toughness were diminished because cubic zirconia does not undergo stress-induced transformation. ${ }^{(4)}$ The most translucent 5Y-PSZ materials were indicated for broad usage as anterior crowns. However, a study revealed a failure rate $>2 \%$ over 5 years in the anterior zone. Applications in minimally invasive restorations, such as veneers, inlays, and onlays, remain to be evaluated. Accordingly, appropriate caution needs to be exercised in placement of this class of zirconia restorative ceramic. ${ }^{(5)}$

\section{Preparation taper}

The preparation taper (convergence angle) refers to the angle between two opposing prepared axial surfaces and is an important feature which gives the prepared tooth its retention and resistance form.

As far back as 1923, Prothero recommended that a taper of $2-5^{\circ}$ was the optimum when preparing a tooth for a crown. ${ }^{(6)}$ However, this was not subjected to scientific studies until the 1950s when Jorgenson used different taper angles and tested the retention of crowns when tensile forces were applied the results supported the earlier $2-5^{\circ}$ recommendation by Prothero. ${ }^{(7)}$ Although this was recommended, clinically it is very difficult to achieve and the reported mean convergence angle produced by dentists ranges between $12^{\circ}$ and $27^{\circ} .{ }^{(8)}$ It has also been found that when molar teeth are prepared they have greater preparation taper compared with the anterior and premolar teeth which is probably due to the greater difficulty with access and trying to avoid damaging the adjacent teeth. ${ }^{(9)}$

In 2004, convergence angles ranging from $0^{\circ}$ to $70^{\circ}$ were studied and the optimum retention being obtained was between $2^{\circ}$ and $20^{\circ}$ with peak retention at $10^{\circ} .^{(10)}$ 


\section{Retention}

Retention is defined as that which prevents the dislodgment of a restoration along the path of insertion or long axis of the tooth preparation, whereas Resistance is the prevention of dislodgment of the restoration by oblique or horizontal forces.

For a crown to resist dislodgment, it is important to have an adequate occlusal-cervical dimension in relation to the preparation taper. There is a linear relationship between the preparation taper and the resistance to dislodgment and this is considered as providing the primary retention or resistance form to a crown preparation. . ${ }^{(11)}$

However, the adhesive bond strength between the restoration and the prepared tooth is a very important factor in the retention and stability of the restoration. This adhesive bond must be strong enough to resist the expected functional loads. Although conventional cementation of zirconium oxide restorations with traditional luting agents (such as zinc-phosphate or resin-modified glass ionomer cements) may provide adequate clinical fixation, adhesive cementation is preferable for ensuring better retention and marginal adaptation. ${ }^{(12)}$

Cement selection is a prerequisite for ensuring effective bond strength to zirconia. Phosphate monomer-based luting agents have been proposed for cementation. ${ }^{(13)}$ such as 10-MDP containing luting systems Panavia v5 with clearfil primer plus (Panavia, Kurary). Self-adhesive cements that rely on a single step application, have also been proposed for luting zirconium-based restorations. (14) The resin matrix of these systems consists of multifunctional acid methacrylates that should react with the substrate and contribute to the adhesion mechanism. ${ }^{(15)}$
Retention of crowns can be measured using universal testing machines where attachments are added to the crowns to which loops are engaged and the crowns are pulled off along the path of insertion. ${ }^{(16)}$

The aim of this study was determining the retention of both adhesive and self-adhesive dualcured cements to cubic zirconia crowns with $12^{\circ}$ and $20^{\circ}$ occlusal convergence preparations.

\section{MATERIALS AND METHODS}

\section{Construction of ceramic samples}

Two stainless steel master dies were prepared by a milling machine to simulate all ceramic full coverage crown preparation of a maxillary premolar. Each Steel die was machined with height $5.5 \mathrm{~mm}$ and two planes occlusal reduction with palatal cusp bevel. The external diameter of the cylinder was $7.5 \mathrm{~mm}$. one die was prepared with $12^{\circ}$ and the other with $20^{\circ}$ occlusal convergence angle. Acrylic bases of $2.5 \mathrm{~cm}$ diameter were fabricated for the dies to aid in the duplication.

\section{Duplication of the master dies}

\section{Duplication fabrication:}

The duplicate of the master dies were taken with a silicone duplicating material * using perforated plastic tubes. Each plastic tube had a diameter of $15 \mathrm{~mm}$ and a height of $30 \mathrm{~mm}$. As recommended by the manufacturer, the two components of the silicone duplicating material were mixed with equal proportions (1: 1) for 30 seconds until uniformly colored dough was obtained. The mixing was done slowly under vacuum to avoid the air trapping then it was poured in the plastic tubes seated on the metal dies for 45 minutes until the material was completely set. Then the impressions were poured to produce epoxy resin dies.

* Dupliflex, Protechno, Spain 


\section{Epoxy resin dies fabrication:}

The impressions of the main master dies were poured using a non-shrink epoxy resin material* . The liquid components A (resin) and B (hardener) were mixed in ratio $2 \mathrm{~A}: 1 \mathrm{~B}$ by weight to a homogenous color for 3 minutes and introduced inside the impressions slowly with a blunted probe on the vibrator to avoid air entrapment. The impressions were removed after 24 hours to ensure that epoxy resin dies reached their maximum hardness.

Dies were distributed according to degree of convergence $\left(12^{\circ}\right.$ group 1$)(n=14)$ and degree of convergence $\left(20^{\circ}\right.$ group 2$)(\mathrm{n}=14)$ and each group was divided into two subgroups according to type of cement (Subgroup A: RelyX Unicem) $(n=7)$ and (Subgroup B: Panavia v5 with clearfil primer plus) $(n=7)$ each die was given a number for identification from one to twenty four.

Intraoral scanner omnicam ${ }^{* *}$ was used to scan dies and produce 28 STL files for the virtual 3D models of the prepared teeth.

The scanned data files were exported as STL files and transferred to the CAD software. Exocad 2017 software ${ }^{* * *}$ was used for designing the restorations. Wings were then added to the proximal surfaces of the crowns for testing the retention.The designed STL files were exported to the CAM software Dental Cam v 6.17.00 **** where the material was selected and the nesting alignment of the crowns in the zirconia blank with buccal and palatal sprues were set.VHF S1 5 -axis milling machine ${ }^{* * * * *}$ was used to mill the crowns. Burs with sizes 2.5, 2, 1 and $0.6 \mathrm{~mm}$ were utilized for milling.
After the milling process all residual dust was removed by placing the restorations in an ultrasonic cleaner filled with fresh distilled water. Restorations were dried first using drying lamp for 30 minutes. The crowns were placed inverted on their occlusal surfaces into the sintering bowl of Nabertherm furnace $\mathrm{P} 310^{* * * * *}$ and sintered at a sintering temperature of $1580^{\circ} \mathrm{C}$.

Clearfil primer plus was applied for the subgroup B 14 crowns then freshly mixed resin cement (Panavia v5) was applied into the fitting surface of the crowns and the other 14 crowns were cemented using Relyx resin cement using clicker auto mix tips to mix and apply the resin cement. The applied cement was then lightly thinned with air to avoid its coagulation. The crowns were placed on their relevant abutments by static finger pressure then axially loaded with a $5 \mathrm{~kg}$ load using a specially designed loading device (7). Crowns were left under the static load for 10 minutes to ensure cement setting. Then crowns were exposed to a brief light curing for only 2 seconds. The excess cement was removed with a scaler, and then light curing was done for 20 seconds for each side.

\section{Measurement of retention}

To test the retention of specimens, Instron universal testing machine was used. A loop chain holding the crown wings to ensure even distribution of pulling tensile forces using a locking mechanism. The cemented crowns were pulled off along the path of insertion with a crosshead speed of $0.5 \mathrm{~mm} /$ minute. The forces required for dislodgment of the crowns were recorded in $\mathrm{N}$.

\footnotetext{
* Kemapoxy 150 , CMB, Egypt

** Dentsply Sirona, USA

*** Exocad GmbH, Darmstadt, Germany

**** VHF, Ammerbuch, Germany

****** VHF, Ammerbuch ,Germany

****** Nabertherm GmbH, Lilienthal, Germany
} 
TABLE (1) List of the main materials used in the study showing their compostion

\begin{tabular}{|c|c|l|c|}
\hline Material & Type & \multicolumn{1}{|c|}{ Composition } & Manufacturer \\
\hline panavia v5 & $\begin{array}{c}\text { Dual polymerizing } \\
\text { resin luting agent }\end{array}$ & $\begin{array}{l}\text { Bisphenol A Diglycidyl-methacrylatw, Triethylene glycol } \\
\text { dimethacrylate, Silanated barium Glass, Silica, Aluminum } \\
\text { oxide, Aromatic dimethacrylate, Aliphatic dimethacrylate, } \\
\text { Camphorquinone, Accelerators, Pigaments }\end{array}$ & $\begin{array}{c}\text { Kura Medical, Inc } \\
\text { Okayama, Japan }\end{array}$ \\
\hline $\begin{array}{c}\text { Clearafil } \\
\text { ceramic } \\
\text { primer plus }\end{array}$ & universal primer & Ethanol, Silane, MDP & $\begin{array}{c}\text { Kurary Medical, Inc } \\
\text { Okayama, Japan }\end{array}$ \\
\hline $\begin{array}{c}\text { RelyX Unicem } \\
\text { Dual polymerizing } \\
\text { resin luting agent }\end{array}$ & $\begin{array}{l}\text { Base/catalyst Methacrylated phosphoric ester, } \\
\text { dimethacrylate, inorganic fillers, fumed silica, chemical } \\
\text { and photoinitiators }\end{array}$ & 3M ESPE, USA \\
\hline
\end{tabular}

Data were statistically analyzed using One-way ANOVA and multiple comparisons were made usingTukey's test at $p<0.05$. SPSS statistical software for windows was used for data analysis.

\section{RESULTS}

One-way ANOVA revealed significant differences between the two groups with the $20^{\circ}$ taper showing lower retention values than the $12^{\circ}$ taper $(\mathrm{P}<0.05)$. It also revealed that the cement type significantly affected the retention strength of the crowns $(\mathrm{P}<0.05)$. The subgroup B showed higher retention force than subgroup A. Mean \pm SD for retention values $(\mathrm{N})$ for subgroups $1 \mathrm{~B}$, and $1 \mathrm{~A}$ were 756 (74) $\mathrm{N}$ and 499 (82) N, respectively. The mean (SD) removal force for subgroups $2 \mathrm{~B}$ and $2 \mathrm{~A}$ were $462(64) \mathrm{N}$ and 305 (76) $\mathrm{N}$, respectively as presented in table no2. Tukey's test indicated that the retention was significantly affected by the occlusal convergence as well as the type of resin cement. Tukey's test revealed that Panavia v5 cement with $12^{\circ}$ taper showed the highest significant retention value, where the lowest value was with RelyX Unicem with $20^{\circ}$ taper.
TABLE (2) Mean \pm SD for retention values (N) for different resin cements with different degrees of convergences
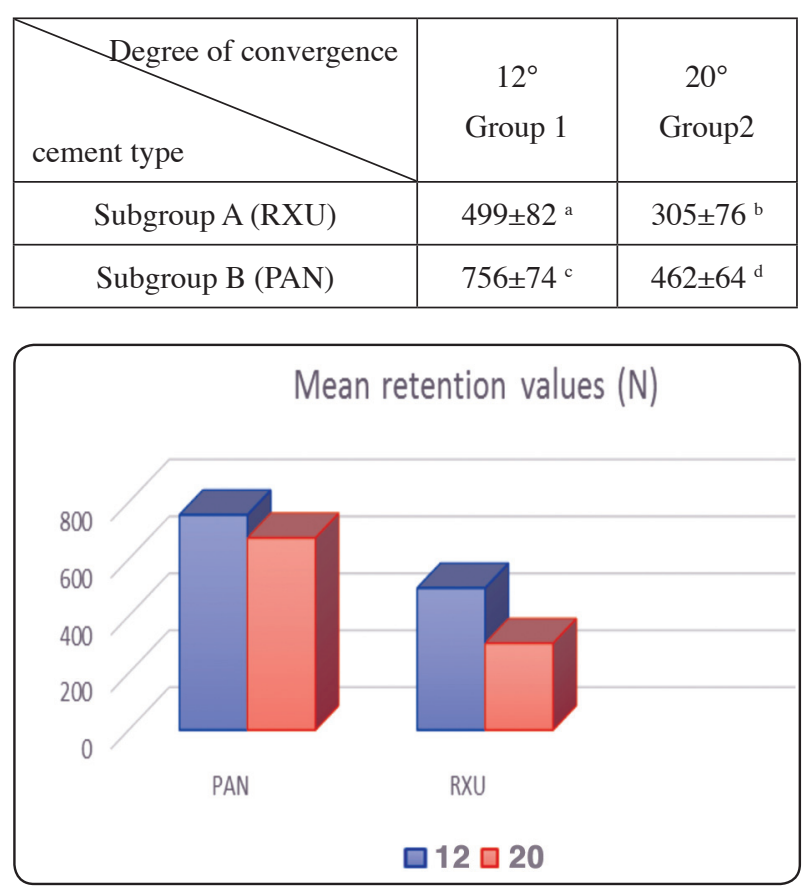

Fig. (1) Bar chart showing mean retention values $(\mathrm{N})$ for different resin cements with different degrees of convergences 


\section{DISCUSSION}

All ceramic restorations have become popular due to esthetic appearance and metal free structure. Zirconia (ZrO2)-based ceramics demonstrates superior mechanical properties such as high fracture strength and fracture toughness. Translucent toothcolored zirconia which enables the fabrication of restorations without using veneering ceramic has been developed. ${ }^{(17)}$ Advantages of monolithic zirconia restorations include limited amounts of defects due to fabrication of the restoration from presintered homogeneous blocks with Computer Aided Design/Computer Aided Manufacturing (CAD/CAM) technique and reduced production time/cost. Also, permitting a minimal material thickness of $0.5 \mathrm{~mm}$ due to high mechanical strength contributes to the preservation of tooth substance and enables the use of ceramic restoration in case of limited interocclusal space. ${ }^{(4)}$

Stainless steel dies were machine milled in the present study to simulate all ceramic full coverage crown preparation of a maxillary premolar and were used as the master dies because of the advantages of standardized preparations, elimination of the errors that may be introduced in the manufacturing process, and lack of wear during fabrication. ${ }^{(18)}$ In the present study each steel die was machined with height $5.5 \mathrm{~mm}$ and $12 \square$ and $20 \square$ total occlusal convergence angle as recommended by Goodacre et al (19) for the preparation of an all-ceramic restoration. An occlusal bevel was prepared at the palatal cusp to help orientation of crowns.

The stainless steel dies were duplicated to avoid scanning using titanium oxide powder that may induce a variable between the intraoral scanning of natural teeth and the intraoral scanner scanning the dies in this study. ${ }^{(20)}$ Epoxy resin material was selected as a die material in the present study on which the test specimens were cemented for investigating their retention since its modulus of elasticity is similar to the reported modulus of human dentin and they are easily available and their dimensions can be standardized. ${ }^{(21)}$

The use of extracted natural teeth as specimens has been found to simulate clinical conditions more closely than resin abutments. However, standardization of natural teeth is difficult because of several factors such as age, anatomy, size, shape and storage time after extraction. ${ }^{(22)}$

Omnicam intraoral scanner was selected cause it's one of the most commonly used intraoral scanners. ${ }^{(23)}$ The updated software cerec 4.5 permits exporting scanned data in STL format to be further used with the CAD software Exocad 2017 for standardizing the design parameters for all samples.

The parameters set for the designed crowns were standardized for all. In this study the cement space was set to $40 \mathrm{um}$, this allows for sufficient flow and and strength of resin cements that have average film thickness of 25-35um. Increasing cement gap to higher values resulted in increased internal misfit and decreasing cement strength. ${ }^{(24)}$ chamfer finish line of $1 \mathrm{~mm}$ thickness ${ }^{(25)}$ and added wings for measuring the retention. ${ }^{(16)}$

During crowns milling, the shrinkage that occurs during the sintering of pre-sintered translucent zirconia blocks is compensated by milling in an enlarged scale corresponding to the sintering parameters defined for this zirconia batch in order not to compromise the adaptation of crowns due to sintering shrinkage. ${ }^{(26)}$

To test the retention of specimens, a universal testing machine was used. A specially customized chain was made to ensure even distribution of pulling tensile forces using a locking mechanism. The cemented crowns were pulled off along the path of insertion with a crosshead speed of $0.5 \mathrm{~mm} /$ minute. The forces required for dislodgment of the crowns were recorded in N. ${ }^{(16)}$

Retention of crowns constructed over dies with $12^{\circ}$ convergence angle showed significantly 
higher retention than those over $20^{\circ}$. This could be attributed to the increased friction and increased surface area for less taper prepared dies.

The findings come in agreement with A. Sarafianou et al. ${ }^{(27)}$ who evaluated the effect of the convergence angle of tooth preparations on retention of resin-bonded restorations and found that increased tapering of tooth preparations resulted in loss of retention and this variable was statistically significant for both cements used when convergence angles exceeded 10 degrees.

Zidan et al. ${ }^{(28)}$ also evaluated the retention of full crowns prepared with 3 different tapers and cemented with 2 conventional and 2 adhesive resin cements. Increasing the taper of the preparation from 6 degrees to 12 degrees did not affect the retention of crowns within the different cement groups. Increasing the taper to 24 degrees decreased the retention of crowns significantly.

And Beuer et al. ${ }^{(29)}$ also studied the effect of different preparation angles $\left(4^{\circ}, 8^{\circ}\right.$, and $\left.12^{\circ}\right)$ on zirconia crowns in relation to marginal and internal fit; here, $12^{\circ}$ was recommended as the best angle for full ceramic crowns. On balance $12{ }^{\circ}$ would appear to be the most appropriate and realistic minimum preparation taper achieved.

Luting cement selection seems to be a relevant factor when bonding to zirconium oxide ceramics. The adhesiveness of phosphate monomer-containing cements increased bond strength, revealing the capability of acidic functional monomers of reacting with the substrate. The application of MDP-containing silane/cement system attained the best overall results. The adhesive potential of 10-MDP to densely sintered zirconia may depend on the presence of a passive coating of zirconium oxide on the ceramic surface. Chemical reactions involving the hydroxyl groups of the layer and the phosphate ester monomers of the MDP occur at the interfacial level. ${ }^{(30)}$ Moreover, the functional monomer has been rated as relatively hydrolysis stable, due to the presence of a long carbonyl chain. A relatively strong poly-molecular layer may be responsible of the ceramic-resin cement bond. ${ }^{(31)}$ However, the longevity of these interfaces should be further evaluated.

RelyX Unicem showed the capability of bonding the substrate. Lower bond strengths were attained if compared to the 10-MDP based cement. Bonding mechanism of RelyX Unicem is reminiscent of the self-adhesiveness of glass ionomer cements and a possible improvement in bond strength may occur after cement maturation overtime. ${ }^{(32)}$

Ernst et al ${ }^{(33)}$ examined the effect of several resin cements, including Unicem and Panavia, on the retentive strength of zirconia crowns fabricated by using the LavaTM system* . The authors found no significant difference in the bond strengths of Unicem and Panavia cements. ${ }^{(33)}$ While significant differences were found by Palacios et al ${ }^{(34)}$ after evaluating the Procera ${ }^{\circledR}$ zirconia. Also, in a recent study done by Layla et al comparing between RelyX Unicem and Panavia F2.0 in cementation of zirconia crowns. The retention values were significantly different. ${ }^{(35)}$ Principally, the retentive bond strength of a crown could be affected by the geometry of the preparation and by the choice of luting agents

Only the early bonding ability of a resin cement to zirconia ceramic was investigated in the present study. The early bond strength of resin cement is quite important because unfavorable clinical situations such as debonding and fractures of ceramic restorations usually occur during or soon after the setting process. If resin cement could produce sufficient bonding to the tooth as well as ceramic restorations soon after setting, the ceramic restoration would in effect be part of the tooth,

* 3M ESPE AG, Seefeld, Germany 
and, as a result, the occurrence of debonding and fractures would be decreased. Of course, the effect of aging on bonding materials should also be taken into consideration and investigated.

\section{CONCLUSIONS}

Within the limitations of this study, the following conclusions were drawn:

1. Retention of ultra-translucent zirconia ceramic differed significantly depending on luting agents and occlusal convergence.

2. The phosphate monomer-containing luting system (Panavia v5 with clearfil primer plus) showed superior results and seems to be the most suitable to bond zirconia ceramic surfaces if compared to self-adhesive resinous cement (RelyX Unicem). However, the durability of these ceramic-to-composite chemical bonds should be further evaluated.

3. Decreasing occlusal convergence to $12^{\circ}$ showed better retention than $20^{\circ}$.

\section{REFERENCES}

1. Guazzato M, Albakry M, Ringer SP, Swain MV. Strength, fracture toughness and microstructure of a selection of all ceramic materials. Part II. Zirconia-based dental ceramics. Dent Mater 2004;20:449-56.

2. Aboushelib MN, Kleverlaan CJ, Feilzer AJ. Microtensile bond strength of different components of core veneered all-ceramic restorations: Part II: Zirconia veneering ceramics. Dent Mater 2006;22:857-63.

3. Tong, H., Tanaka, C. B., Kaizer, M. R. \& Zhang, Y. Characterization of three commercial Y-TZP ceramics produced for their high-translucency, high-strength and high-surface area. Ceram. Int.2016; 42, 1077-1085.

4. Zhang, F. et al. Strength, toughness and aging stability of highly-translucent Y-TZP ceramics for dental restorations. Dent. Mater.2016; 32, e327-e337.

5. Sulaiman, T. A., Abdulmajeed, A. A., Shahramian, K. \& Lassila, L. Effect of different treatments on the flexural strength of fully versus partially stabilized monolithic zirconia. J. Prosthet. Dent. 2017;118, 216-220.
6. Goodacre, C. J., Campagni, W. V. \& Aquilino, S. A. Tooth preparations for complete crowns: an art form based on scientific principles. J. Prosthet. Dent.2001; 85, 363-76.

7. Jorgensen, K. D. The relationship between retention and convergence angle in cemented veneer crowns. Acta Odontol. Scand. 1955;13, 35-40.

8. Smith, C. T., Gary, J. J., Conkin, J. E. \& Franks, H. L. Effective taper criterion for the full veneer crown preparation in preclinical prosthodontics. J. Prosthodont. 1999;8, 196-200

9. Annerstedt, A. et al. Axial wall convergence of full veneer crown preparations. Documented for dental students and general practitioners. Acta Odontol. Scand. 1996;54, 109-12.

10. CHAN, D. C. N. et al. Effect of preparation convergence on retention and seating discrepancy of complete veneer crowns. J. Oral Rehab.2004;31, 1007-1013.

11. Blair, F. M., Wassell, R. W. \& Steele, J. G. Crowns and other extra-coronal restorations:Preparations for full veneer crowns. Br. Dent. J. 2002;192, 561-571.

12. Derand T, Molin M, Kvam K. Bond strength of composite luting cement to zirconia ceramic surfaces. Dent Mater 2005;21:1158-62.

13. Kern M, Wegner SM. Bonding to zirconia ceramic: adhesion methods and their durability. Dent Mater 1998;14:64-71.

14. Kumbuloglu O, Lassila LVJ, User A, Vallitu PK. Bonding of resin composite luting cements to zirconium oxide by two air-particle abrasion methods. Oper Dent 2006;31:248-55.

15. De Munck J, Vargas M, Van Landuyt K, Hikitaa K, Lambrechts P, Van Meerbeek B. Bonding of an auto-adhesive luting materials to enamel and dentin. Dent Mater 2004;20:963-71.

16. Aleisa, K. et al. Retention of zirconium oxide copings using different types of luting agents. J. Dent. Sci.2013; 8, 392-398.

17. Raigrodski AJ, Hillstead MB, Meng GK, Chung KH. Survival and complications of zirconia-based dental prostheses: a systematic review. J Prosthet Dent. 2012;107(3):170-7.

18. Gonzalo, E., Suárez, M. J., Serrano, B. \& Lozano, J. F. L. A comparison of the marginal vertical discrepancies of zirconium and metal ceramic posterior fixed dental prostheses before and after cementation. J. Prosthet. Dent.2009; $102,378-384$. 
19. Goodacre, C., Campagni, W. \& Aquilino, S. Tooth preparation for complete crowns: an art form base on schientific principles. J Prosthet Dent .2001;85, 363-376.

20. Lee, W., Kim, W., Kim, H., Kim, W. \& Kim, J. Evaluation of different approaches for using a laser scanner in digitization of dental impressions.2014 Feb; 6(1): 22-29.

21. Rosentritt, M., Plein, T., Kolbeck, C., Behr, M. \& Handel, $\mathrm{G}$. In vitro fracture force and marginal adaptation of ceramic crowns fixed on natural and artificial teeth. Int. J. Prosthodont. 2000: 13, 387-91.

22. Alghazzawi, T. F., Lemons, J., Liu, P.-R., Essig, M. E. \& Janowski, G. M. The failure load of CAD/CAM generated zirconia and glass-ceramic laminate veneers with different preparation designs. J. Prosthet. Dent.2012; 108, 386-393.

23. Ebeid, K., Salah, T. \& Nossair, S. Accuracy and Reliability of Intraoral Scanners : Are They the Better Option? Dent. Restor. Mater.2017; 209-214.

24. Iwai, T., Komine, F., Kobayashi, K., Saito, A. \& Matsumura, H. Influence of convergence angle and cement space on adaptation of zirconium dioxide ceramic copings. Acta Odontol. Scand. 2008;66, 214-218.

25. Henrique, $P$. et al. Influence of convergence angle of tooth preparation on the fracture resistance of Y-TZP-based allceramic restorations. Dent. Mater.2013; 29, 339-347.

26. ABDUO, J., LYONS, K. \& SWAIN, M. Fit of zirconia fixed partial denture: a systematic review. J. Oral Rehabil.2010; 37, 866-876.

27. Sarafianou, A. \& Kafandaris, N. M. Effect of convergence angle on retention of resin-bonded retainers cemented with resinous cements. J. Prosthet. Dent. 1997;77, 475-81.

28. Zidan, O. \& Ferguson, G. C. The retention of complete crowns prepared with three different tapers and luted with four different cements. J. Prosthet. Dent.2003; 89, 565-71.

29. Beuer, F. et al. Influence of preparation angle on marginal and internal fit of CAD / CAM-fabricated zirconia crown copings. Quintessence Int. 2009;40, 243-250.

30. Derand T, Molin M, Kvam K. Bond strength of composite luting cement to zirconia ceramic surfaces. Dent Mater 2005;21:1158-62.

31. Berry T, Barghi N, Chung K. Effect of water storage on the silanization in porcelain repair strength. J Oral Rehab 1999; 26:459-63.

32. Roulet JF, Soderholm KJ, Longmate J. Effects of treatment and storage conditions on ceramic/composite bond strength. J Dent Res 1995;74: 381-7.

33. Ernst CP, Cohnen U, Stender E, Willershausen B. In vitro retentive strength of zirconium oxide ceramic crowns using different luting agents. J Prosthet Dent. 2005 Jun; 93(6):551-8.

34. Palacios RP, Johnson GH, Phillips KM, Raigrodski AJ. Retention of zirconium oxide ceramic crowns with three types of cement. J Prosthet Dent. 2006 Aug;96(2):104-14.

35. Leyla Sadighpour, Farideh Geramipanah, Akbar Fazel, Mahdi Allahdadi and Mohammad Javad Kharazifard.Effect of Selected Luting Agents on the Retention of CAD/ CAM Zirconia Crowns Under Cyclic Environmental Pressure.J Dent (Tehran). 2018; 15(2): 97-105. 